Research Paper

\title{
A nomogram based on phosphorylated AKT1 for predicting locoregional recurrence in patients with oesophageal squamous cell carcinoma
}

\author{
Weiwei $\mathrm{Yu}^{1,2^{*}}$, Li Chu ${ }^{1,5^{*}}$, Kuaile Zhao ${ }^{1,5}$, Haiquan Chen ${ }^{3,5}$, Jiaqing Xiang3,5, Yawei Zhang ${ }^{3,5}$, Hecheng $\mathrm{Li}^{3,5}$, \\ Weixin Zhao ${ }^{1,5}$, Menghong Sun ${ }^{4,5}$, Qiao Wei ${ }^{4,5}$, Xiaolong Fu' ${ }^{1,6}$, Congying Xie ${ }^{7}$ and Zhengfei Zhu ${ }^{1,5}$ \\ 1. Department of Radiation Oncology, Fudan University Shanghai Cancer Center, Shanghai, China; \\ 2. Department of Radiation Oncology, Shanghai Jiao Tong University Affiliated Sixth People's Hospital, Shanghai, China; \\ 3. Department of Thoracic Surgery, Fudan University Shanghai Cancer Center, Shanghai, China; \\ 4. Department of Pathology, Fudan University Shanghai Cancer Center, Shanghai, China; \\ 5. Department of Oncology, Shanghai Medical College, Fudan University, Shanghai 200032, China; \\ 6. Department of Radiation Oncology, Shanghai Chest Hospital, Shanghai Jiao Tong University, Shanghai, China; \\ 7. Radiotherapy and Chemotherapy Department, the 1st Affiliated Hospital of Wenzhou Medical University, Wenzhou, China \\ *Weiwei Yu and Li Chu contributed equally to this work. \\ $\square$ Corresponding author: Correspondence: Professor Zhengfei Zhu, Department of Radiation Oncology, Fudan University Shanghai Cancer Center, Shanghai, \\ China; E-mail: fuscczzf@163.com. \\ (c) Ivyspring International Publisher. This is an open access article distributed under the terms of the Creative Commons Attribution (CC BY-NC) license \\ (https://creativecommons.org/licenses/by-nc/4.0/). See http://ivyspring.com/terms for full terms and conditions.
}

Received: 2017.05.02; Accepted: 2017.08.30; Published: 2017.10.17

\begin{abstract}
Background: The AKT signalling pathway controls survival and growth in many malignant tumours. However, the prognostic value of phosphorylated AKT1 (p-AKTl) for locoregional-progression free survival (LPFS) in oesophageal squamous cell carcinoma (ESCC) has not been established. Our aim was to develop a nomogram to predict local recurrence using $\mathrm{P}-\mathrm{AKT1}$ and main clinical characteristics in patients with thoracic ESCC undergoing radical three-field lymph node dissection.

Methods: Immunohistochemistry was performed to examine p-AKTl expression in 181 thoracic ESCC patients. The Kaplan-Meier method was used to calculate LPFS. Cox regression analysis was also performed to evaluate prognostic factors. A nomogram comprising biological and clinical factors was established to predict LPFS.

Results: The 5-year LPFS rate was $63.9 \%$. Multivariate analysis revealed that expression of $p$-AKT1 $(p<0.001)$, pathologic $N$ category $(p=0.004)$ and number of lymph nodes retrieved $(p=0.001)$ were independent prognostic factors for LPFS. Increased expression of $p-A K T 1$ was associated with decreased LPFS in patients with ESCC. In addition, a nomogram was established based on all significant independent factors for locoregional recurrence risk. Harrell's c-index for predicting LPFS was 0.78 .
\end{abstract}

Conclusion: Activation of AKTI was associated with poor locoregional control in ESCC patients. The nomogram, based on p-AKTl expression and clinically significant parameters, could be used as an accurate stratification model for predicting locoregional recurrence in patients with ESCC after radical resection.

Key words: Esophageal squamous cell carcinoma, Locoregional-progression free survival, Phosphorylated AKT1, Nomogram, Prognostic factor

\section{Introduction}

Despite advances in surgical techniques and improvements in perioperative management, current postoperative prognosis for patients with oesophageal squamous cell carcinoma (ESCC) 
remains discouraging [1-2]. The main reason for postoperative failure is locoregional recurrence. Even after radical oesophagectomy with extensive three-field lymph node dissection (3FLD), the locoregional recurrence rate is as high as $40 \%$ [3-4]. Several randomized trials have evaluated the role of more aggressive local treatment, surgery combined with postoperative radiotherapy (PORT) with or without chemotherapy, which is one of the important strategies that increases locoregional control [5-6].

However, the use of PORT for the improvement of local control and overall survival (OS) has been controversial [7-8]. It is at least partially due to the lack of efficient criteria for predicting locoregional recurrence and determining which patients would more likely benefit from PORT. In current clinical practice, we mostly rely on clinicopathological characteristics, such as pathological tumour-node-metastasis (pTNM) staging, to stratify patients for improving treatment after surgery [9]. Nevertheless, tumour biology may not always reflect clinicopathological features [10-11]. Clearly, identifying effective biomarkers to refine the current stratification model for predicting locoregional recurrence in patients with ESCC is of great importance.

In the previous study, we investigated the influence of expression of epidermal growth factor receptor (EGFR) signalling pathway-related proteins on OS in patients with ESCC. A panel of 11 biomarkers was chosen for immunohistochemical analysis, including EGFR, phosphorylated (p)-EGFR, AKT1, AKT2, ERK1, ERK2, STAT3, p-AKT1, p-AKT2, p-ERK1/2, and p-STAT3. We found that expression of p-AKT1 was an independent prognostic factor for OS, with higher expression of p-AKT1 associated with poorer OS [12]. The purpose of the current study was to evaluate the role of p-AKT1 expression combined with other clinical factors, using a nomogram, in predicting locoregional recurrence in patients with ESCC after radical oesophagectomy and guiding tailored postoperative radiotherapy strategy.

\section{Materials and methods}

\section{Study population}

A series of studies has been conducted to explore clinical and biological prognostic factors in patients with thoracic ESCC who underwent a radical oesophagectomy with three-field lymphadenectomy (3FLND) at Fudan University Shanghai Cancer Centre between 2001 and 2009 [12-14]. The primary inclusion criteria for these studies were as follows: (1) confirmed ESCC by histopathology, and classified according to the seventh edition of the
TNM-UICC/AJCC classification system [15]; (2) having only one primary tumour; (3) not receiving preoperative chemotherapy and/or radiotherapy; (4) having undergone 3FLND with $\geq 15$ total lymph nodes removed; and (5) having $\mathrm{R} 0$ resection.

The aim of this study was to investigate the predictive value of biological and clinicopathological factors on locoregional recurrence. To eliminate the confounding influence of local treatment after surgery on LPFS, patients who had accepted postoperative radiotherapy and chemoradiotherapy were excluded; patients whose postoperative treatments were unknown were also excluded. As a result, 249 patients were selected. Of these patients, 24 were excluded from analysis because of perioperative deaths (two patients) and loss to follow-up related to LPFS (22 patients). Among the 225 remaining patients, paraffin specimens were not available for 44 of them. Finally, a total of 181 patients were included in the study.

The preoperative workup, surgical procedure and follow-up were described elsewhere [13-14]. Locoregional failure included oesophagus, tumour bed, anastomotic stoma and regional lymph node recurrences. If an oesophageal or anastomotic stoma recurrence was suspected, confirmation was obtained using endoscopy and a biopsy. Regional lymph nodes were defined as extending from periesophageal cervical nodes to celiac nodes, according to the AJCC (7th edition) staging system. Therefore, recurrence in lymph nodes in these areas was considered to be regional recurrence in this study. Regional lymph node recurrence was defined as an increase in the lymph node size on successive follow-up CT-scans, and for some patients, suspected lymph node recurrences were evaluated using PET and/or a biopsy.

\section{Immunohistochemistry (IHC) and expression evaluation}

Tissue microarrays (TMAs) were constructed as previously described [12]. IHC staining was performed on 4- $\mu \mathrm{m}$ paraffin-embedded sections from TMAs using the standard Envision method with a primary antibody of p-AKT1 (Ser473) (EP2109Y, dilution 1:100; Abcam) (Fig. 1).

Tissue sections were assessed independently in a blinded manner by three histopathologists. Discrepancies were resolved through consensus. A modified semiquantitative $\mathrm{H}$-score method was used to evaluate IHC staining [16]. Staining in either the cytoplasmic or the nuclear compartment was considered positive. For each tissue core, a score was generated by multiplying the percentage of positive cells $(0-100 \%)$ and the intensity of staining. The staining intensity for the IHC reaction was classified 
as follows: 0 , negative; $1+$, weak; $2+$, moderate; and $3+$, strong. Thus, the overall $\mathrm{H}$-score ranged from $0-300$.

\section{Statistical analysis}

Statistical evaluation was conducted using SPSS17.0 (SPSS Inc., Chicago, IL, USA) and R 3.3.1 software (Institute for Statistics and Mathematics, Vienna, Austria). LPFS was calculated from the date of surgery to documented locoregional recurrence, or the last visit, which was censored as of the date of the last follow-up. Patients who died without locoregional recurrence were censored at the date of death. Survival curves were constructed using the Kaplan-Meier method and compared using the log-rank test to evaluate the statistical significance of differences. Since there's no standard cut-off points for the p-AKT1 at present, we chose the median $\mathrm{H}$-score values as the cut-off points for the categorical analyses: high expression was defined as an H-score > the median value, and low expression as an $\mathrm{H}$-score $\leq$ the median value. Univariate and multivariate Cox regression analyses were performed to test for the independent influence of potential prognostic factors on LPFS. $P<0.05$ was considered statistically significant, and statistical tests were based on a two-sided significance level. A nomogram based on possible prognostic factors associated with LPFS was established using $\mathrm{R}$ software. The performance of the nomogram was assessed with respect to

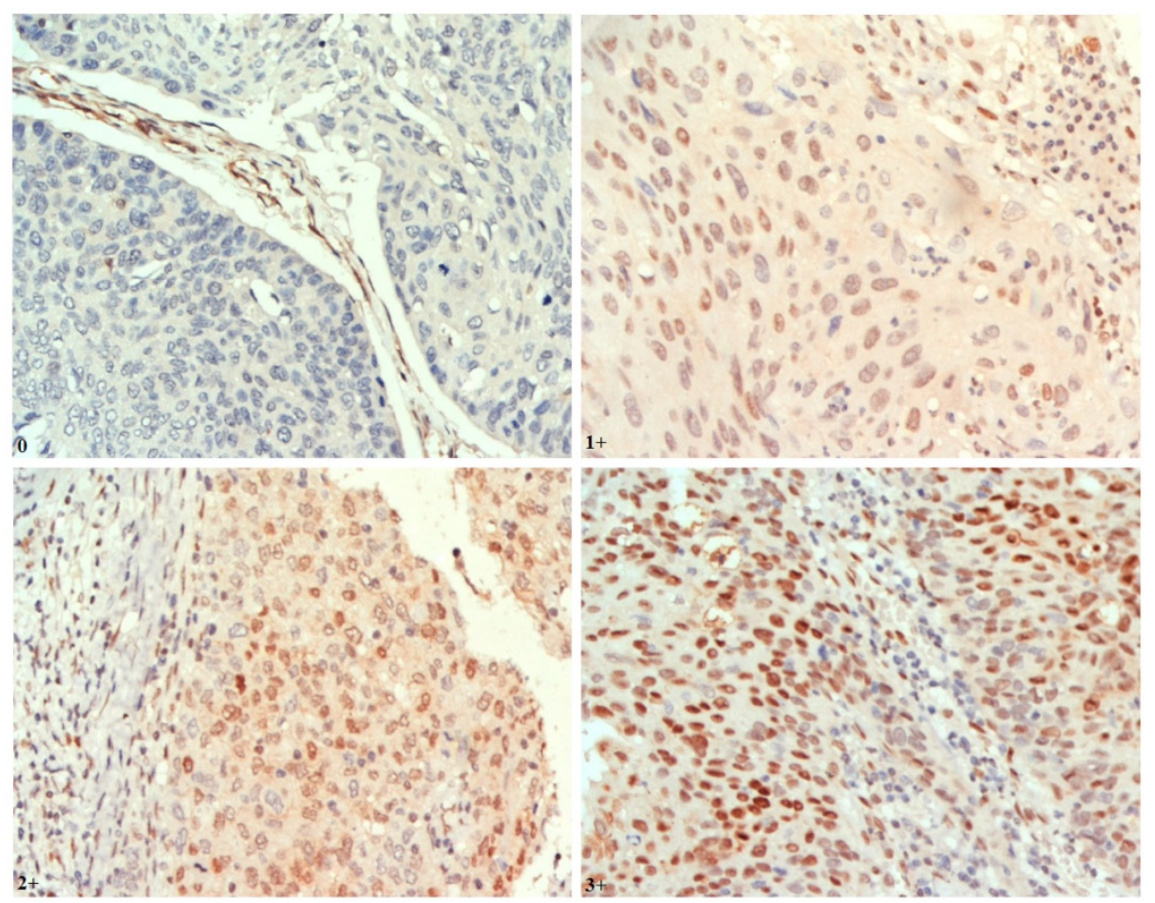

Fig 1. Representative findings on immunohistochemistry analysis of p-AKTl expression in oesophageal squamous cell carcinoma $(E S C C)$ (nuclear staining, magnification $\times 400$ ) Staining: 0 , negative; $1+$, weak; $2+$, moderate; $3+$, strong positivity. discrimination and calibration. To reduce the overfit bias, we used the bootstrap technique with 160 repetitions for internal validation. Discrimination was evaluated with Harrell's concordance index (C-index). The C-index estimates the probability of concordance between the observed LPFS and LPFS that are predicted from the model. The value of the C-index statistic ranged from 0.5 (no discrimination) to 1 (perfect discrimination), with 1 indicating perfect concordance, 0.5 indicating no better concordance than chance, and higher C-index values indicated a better prognostic model [17-18]. Calibration was quantified by comparing the predicted LPFS with that of the observed survival against the nomogram's 1-, 3and 5-year predicted LPFS probabilities.

\section{Results}

\section{Patient characteristics}

Clinical characteristics of patients are summarized in Table 1 . The median age was 58 years (range 38-78 years), and most patients were male $(81.8 \%)$. The majority of cases $(63.5 \%)$ occurred in the middle thoracic oesophagus. Most tumours (68.5\%) showed moderately differentiated histological grades. Of the 181 studied patients, 80 received postoperative chemotherapy. Of the 59 patients who experienced locoregional recurrence, 35 did not receive any treatment after surgery.

\section{Patterns of locoregional recurrence}

Ninety-one patients $(50.3 \%)$ were recognized treatment failures during the follow-up period, with 53 patients $(29.3 \%)$ experiencing locoregional recurrence alone, 32 patients $(17.7 \%)$ with distant metastases alone and six patients (3.3\%) having both locoregional and distant recurrences. Among the 59 patients with locoregional recurrence, 19 had local site recurrence (13 in the primary oesophageal tumour bed and six in anastomotic stoma); 38 patients $(21 \%) \quad$ developed regional lymph node recurrence (eight in cervical, 18 in thoracic, four in abdominal, and eight in cervical and thoracic sites); two patients $(1.1 \%)$ suffered local site combined with regional lymph node recurrences (Table 2). 
Table 1. Clinicopathological characteristics in ESCC patients $(n=181)$

\begin{tabular}{ll}
\hline Characteristics & No. of patients (\%) \\
\hline Gender & $148(81.8)$ \\
Male & $33(18.2)$ \\
Female & \\
Age (years) & 58 (range 38-78) \\
Median & $104(57.5)$ \\
$<60$ & $77(42.5)$ \\
$\geq 60$ & \\
Tumor location & $18(10)$ \\
Upper & $115(63.5)$ \\
Middle & $48(26.5)$ \\
Lower & \\
Tumor length (cm) & $75(41.4)$ \\
$<5$ & $106(58.6)$ \\
$\geq 5$ & \\
Tumor differentiation & $17(9.4)$ \\
Well & $124(68.5)$ \\
Moderate & $40(22.1)$ \\
Poor & \\
Pathological vascular invasion & $64(35.4)$ \\
Negative & $117(64.6)$ \\
Positive & \\
Pathologic T stage & $6(3.3)$ \\
T1 & $74(40.9)$ \\
T2 & $82(45.3)$ \\
T3 & $19(10.5)$ \\
Pathologic N stage & \\
N0 & $68(37.6)$ \\
N1 & $52(28.7)$ \\
N2 & $40(22.1)$ \\
N3 No. of lymph nodes retrieved & $21(11.6)$ \\
$\leq 20$ & \\
$<20 ~ \leq 30$ & $29(16)$ \\
$>30$ & $72(39.8)$ \\
Adjuvant therapy & $80(44.2)$ \\
None & \\
Chemotherapy & $101(55.8)$ \\
\hline & $80(44.2)$ \\
&
\end{tabular}

Table 2. Site of locoregional recurrence in all patients

\begin{tabular}{lll}
\hline Site of locoregional recurrence & $\mathrm{n}$ & $\%$ \\
\hline Local recurrence & 19 & 10.5 \\
Primary esophageal tumor bed & 13 & 7.2 \\
Anastomotic stoma & 6 & 3.3 \\
Regional lymph nodes recurrence & 38 & 21 \\
Cervical & 8 & 4.4 \\
Thoracic & 18 & 10 \\
Abdominal & 4 & 2.2 \\
Cervical plus Thoracic & 8 & 4.4 \\
Local combined regional lymph nodes recurrence & 2 & 1.1 \\
Total & 59 & 32.6 \\
\hline
\end{tabular}

\section{Prognostic factors for LPFS}

Median follow-up time for all patients was 36.8 months (range 2-127 months). OS and LPFS rates over time are shown in Fig. 2A. The 1-, 3-, 5-year OS and LPFS rates were $84 \%, 56.9 \%, 52.2 \%$ and $82.2 \%, 66.6 \%$, $63.9 \%$, respectively.

The median H-score for p-AKT1 expression was 70 (range: 0-250), which we chose as the cut-off point, and patients were then divided into a low-level expression group (H-scores $\leq 70 ; n=95)$ and a high-level expression group ( $\mathrm{H}$-scores $>70 ; n=86$ ) for further analysis. Variables tested in univariate analysis showed that significant factors associated with LPFS included pathologic $\mathrm{N}$ category $(\mathrm{p}<0.001)$, number of lymph nodes retrieved $(p=0.003)$, pathological vessel invasion $(\mathrm{p}=0.005)$ and expression of p-AKT1 $(p<0.001)$ (Table 3). All variables with statistical significance in univariable analysis were then evaluated in the multivariate Cox proportional hazards model to adjust for effects of covariates.

Multivariate analyses demonstrated that $\mathrm{p}-\mathrm{AKT} 1$ expression $(\mathrm{p}<0.001)$, pathologic $\mathrm{N}$ category $(\mathrm{p}=0.004)$ and number of lymph nodes retrieved $(p=0.001)$ were independent prognostic factors for locoregional recurrence in patients with ESCC (Table 3). The LPFS curves compared using log-rank tests according to $\mathrm{N}$-staging, number of lymph nodes retrieved and expression of p-AKT1 are shown in Fig. 2. Furthermore, we stratified patients according to their lymph node metastasis status, and found that the group with p-AKT1 low expression had significantly better LPFS than the group with p-AKT1 high expression among patients with negative lymph nodes $(p=0.002$, Fig. 3A) and patients with positive lymph nodes ( $p<0.001$, Fig. 3B).

A nomogram incorporating all significant independent factors for predicting locoregional recurrence was established based on selected variables with hazard ratios (Fig. 4). Each variable was given a score on a points scale. By adding up the total scores projected in the bottom scale, we could estimate the probability of 1-, 3- and 5-year LPFS. Based on intern validation, the Harrell's C-index for LPFS prediction was 0.78 (95\% CI, 0.74-0.82). The calibration plot for the probability of LPFS at 1, 3 or 5 years after surgery showed a good correlation between the nomogram prediction and actual observation (Fig. 5).

\section{Discussions}

In this study, we found that locoregional recurrence was the major pattern of treatment failure. Even after extended radical oesophagectomy with 3FLND, the rate of locoregional recurrence was slightly higher than haematogenous metastasis, which was similar to other studies [3, 19]. We also demonstrated that clinical factors including pathologic $\mathrm{N}$ category and number of lymph nodes retrieved, in combination with p-AKT1 expression, could be more valuable in predicting locoregional recurrence, which may more precisely guide postoperative therapy; however, further investigation is needed. 

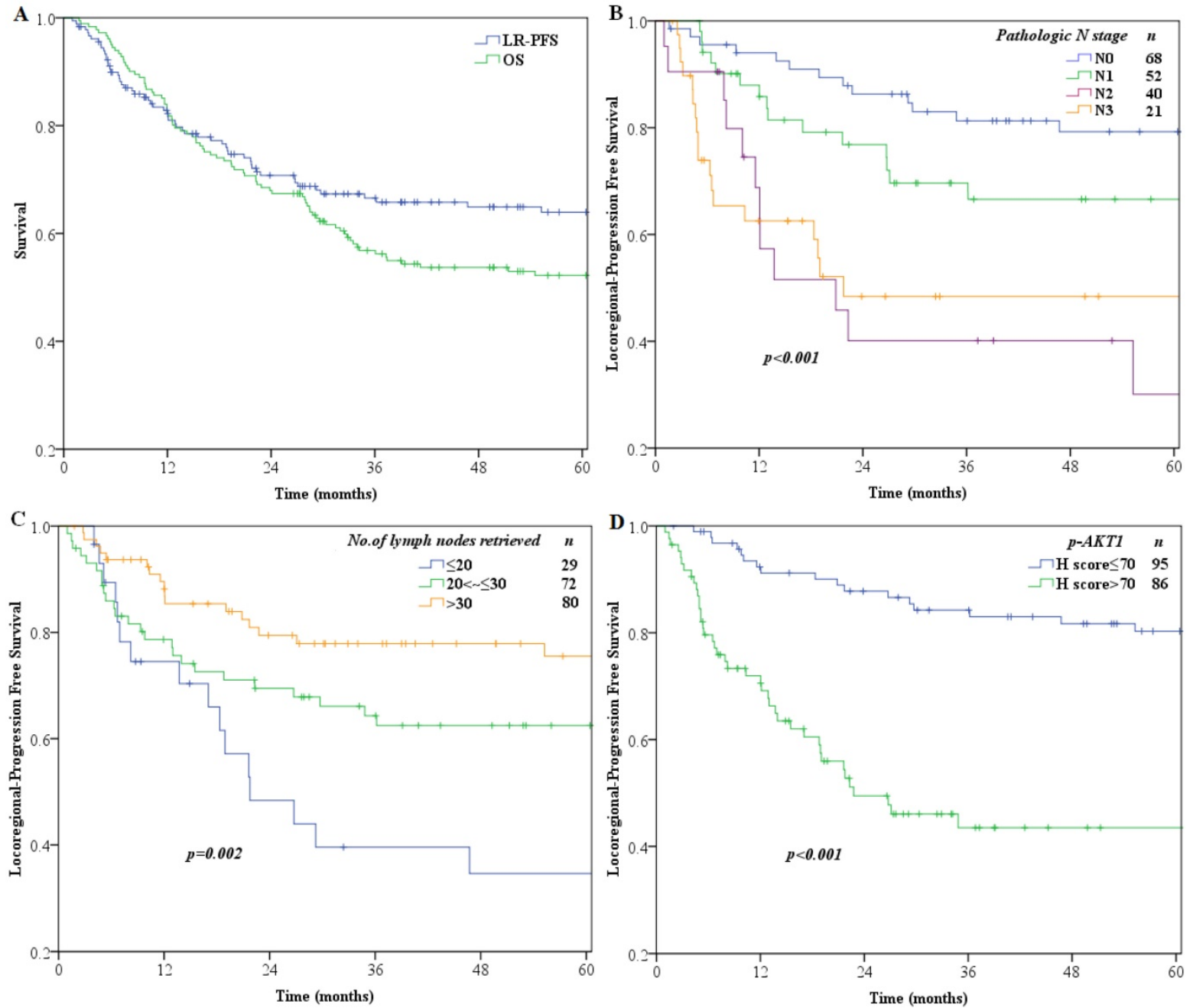

Fig 2. Kaplan-Meier curve analysis of survival (A) OS and LPFS of the entire study population of 181 patients (B) LPFS of patients stratified by pathologic $N$ stage (C) LPFS of patients stratified by number of lymph nodes retrieved (D) LPFS of patients stratified by P-AKTI H-scores.
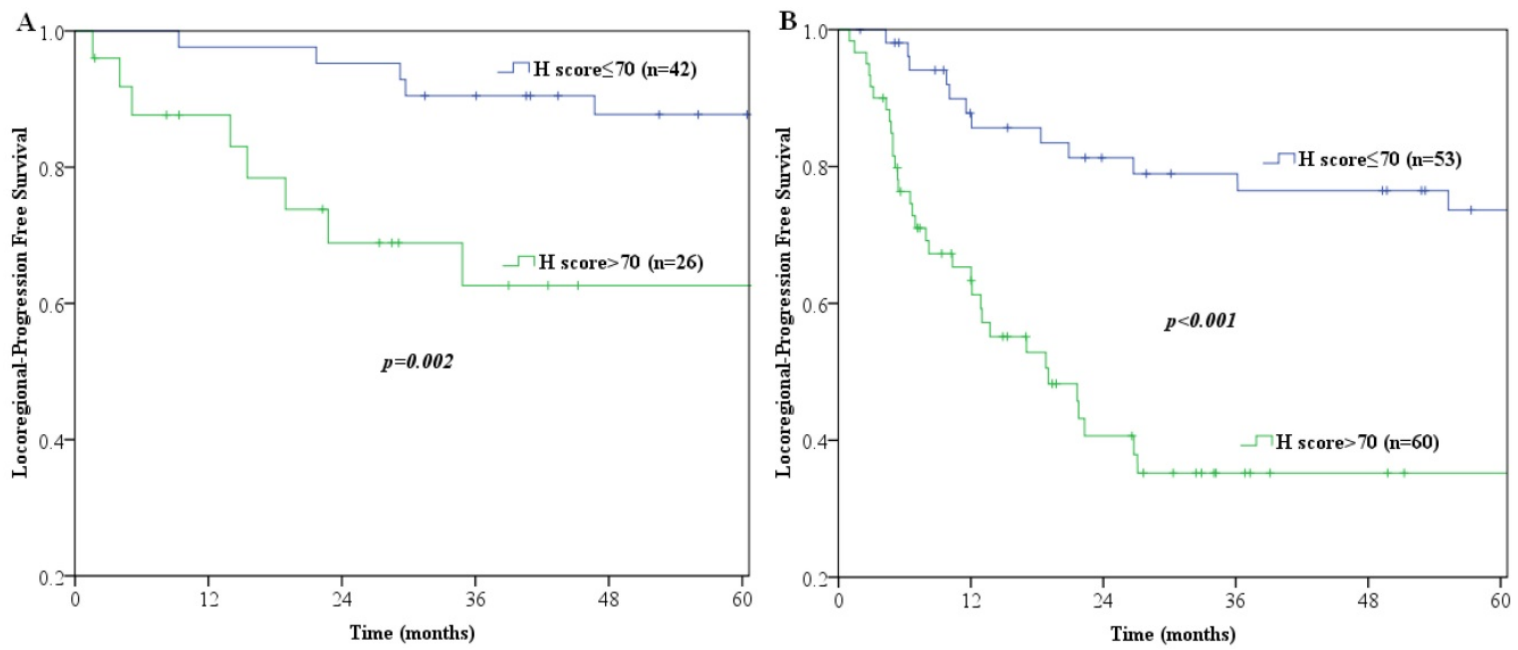

Fig 3. Log-rank tests of LPFS comparing patients with $p$-AKT1 H-scores of $\leq 70$ and those with $p$-AKT1 H-scores of $>70$ for $(A)$ lymph nodes negative patients $(n=68$; $p=0.002)(B)$ lymph nodes positive patients $(n=113 ; p<0.001)$. 

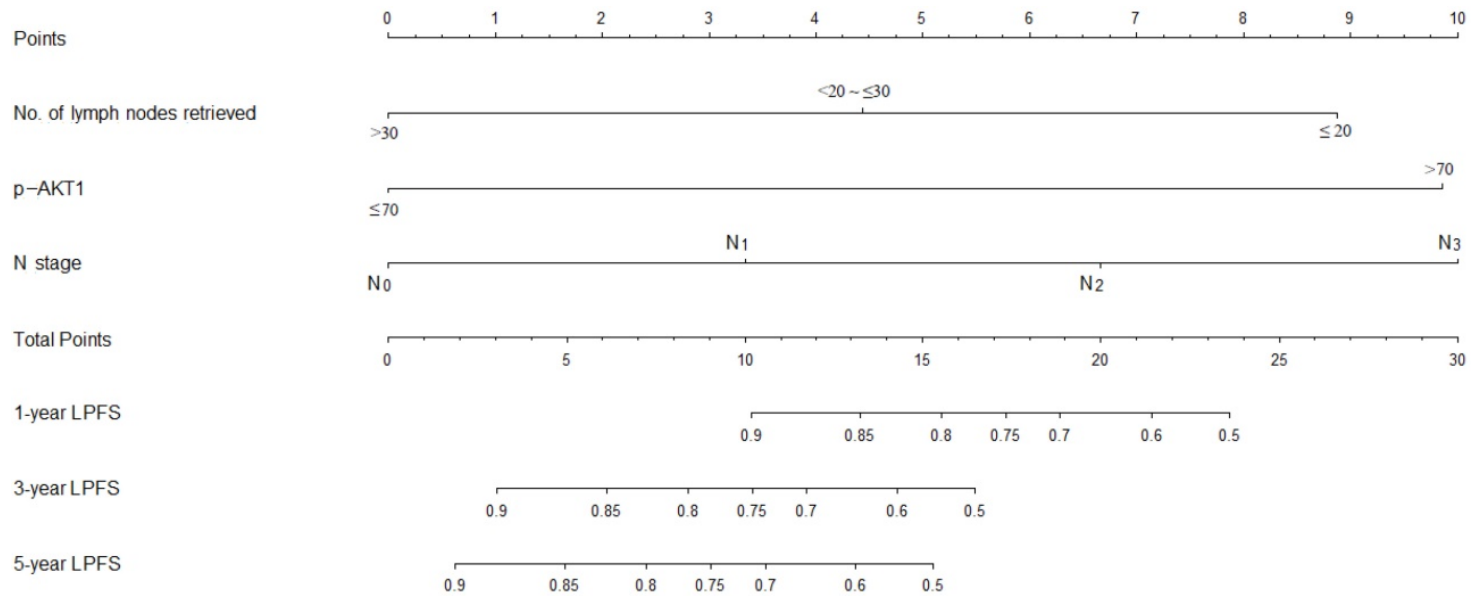

Fig 4. Nomogram predicts LPFS based on expression of p-AKT1 and clinical variables. The nomogram is used by totaling the points identified at the top of the scale for each independent factor. This total point score is then identified on the total points scale to determine the probability of LPFS prediction. The Harrell's c-index for LPFS prediction was 0.78 .
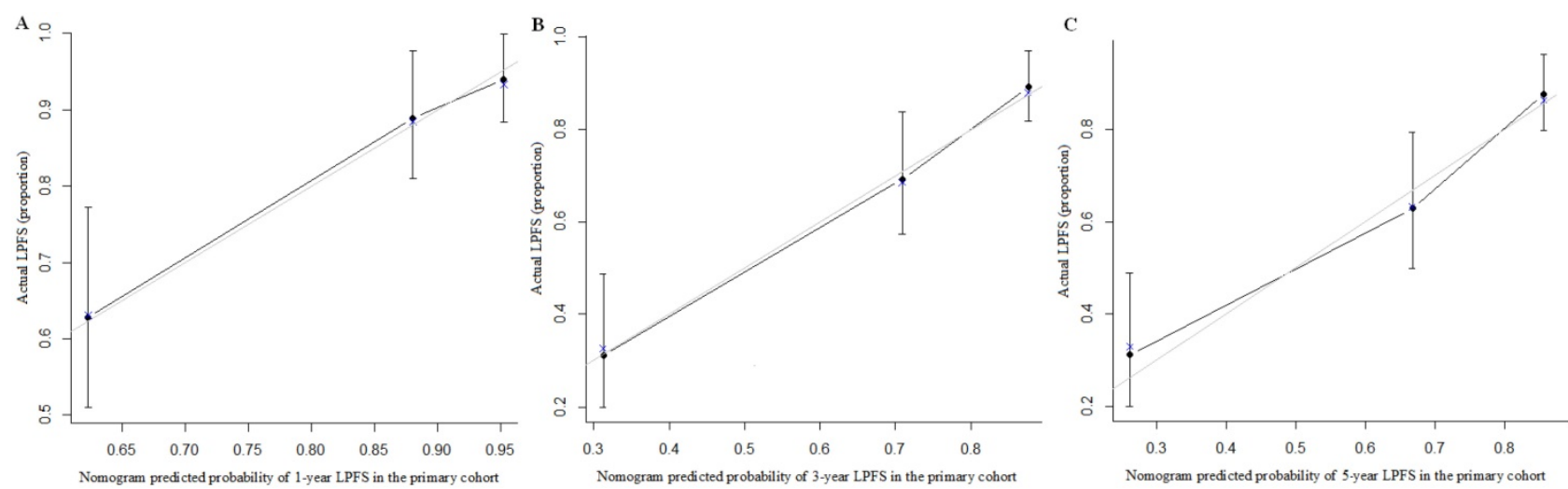

Fig 5. The calibration curves for predicting patient LPFS at (A) 1-year; (B) 3-year and (C) 5-year in the intern validation. Nomogram-predicted locoregion-progression free survival is plotted on the $x$-axis; actual survival is plotted on the $y$-axis.

PI3K/Akt signalling, which is involved in tumourigenesis, is now known to play a pivotal role in the control of metabolism, cell growth, proliferation, survival or migration, and membrane transport or secretion [20]. PI3K phosphorylates membrane-bound phosphatidylinositol diphosphate (PIP2) to generate phosphatidylinositol trisphosphate (PIP3), and thus permits phosphorylation (i.e. activation) of AKT at Thr308 and Ser473 residues in the presence of PDK1 and mTORC2, respectively [21]. Activation of AKT can induce the phosphorylation of procaspase-9, thereby inhibiting its protease activity. It also phosphorylates Bad, a proapoptotic member of the Bcl-2 family, and the forkhead transcription factor FKHR, a proapoptotic transcription factor. Therefore, p-AKT mediates a range of prosurvival signals for regulating apoptosis, proliferation, cell growth and angiogenesis [22-23].

There is increasing evidence that p-AKT1 is significantly overexpressed in several tumours and is associated with a higher risk of disease recurrence [24-25]. Kreisberg et al. [26] found that phosphorylation of AKT1 at the Ser473 residue is an independent prognostic factor in prostate cancer, and positive expression of $\mathrm{p}$-AKT1 protein predicted decreased survival. Ayala et al. [27] reported that high expression of p-AKT1 in prostate cancer was predictive of a higher probability of recurrence. Also, p-AKT1 expression was an independent prognostic indicator of biochemical recurrence-free survival when Gleason 6 and 7 patients were analysed separately.

In the present study, we found a significant association between high-level expression of p-AKT1 and poor LPFS in patients with ESCC after radical surgery. Multivariable Cox regression analysis showed that p-AKT1 expression was strongly and independently predictive of LPFS (hazard ratio $=4.335$, [95\% CI, 2.327-8.076], p<0.001). Moreover, high expression of p-AKT1 predicted decreased LPFS independent of lymph node status. Even in lymph node-negative patients, we could further predict LPFS by detecting p-AKT1 expression in tumour tissues. Expression levels of p-AKT1 might enable 
stratification of locoregional recurrence risk in patients with similar clinicopathological factors. To the best of our knowledge, this is the first study to establish a nomogram based on PI3K/Akt signalling pathways for predicting postoperative LPFS in ESCC. In several previous literature, different models were developed to predict the overall survival or distant metastasis for patients with ESCC after radical oesophagectomy [28-29]. In another study of Hayashi et al.[30], a nomogram combined clinical parameters was developed to predict lymph node metastasis after preoperative chemoradiotherapy, which could be used to select the type of surgical procedure and explain patients about surgical necessity.

Table 3. Univariate and multivariate analyses of LPFS in ESCC patients

\begin{tabular}{|c|c|c|c|c|}
\hline \multirow[t]{2}{*}{ Characteristics } & Univariate analysis & \multirow[t]{2}{*}{$p$ Value } & $\begin{array}{l}\text { Multivariate } \\
\text { analysis }\end{array}$ & \multirow[t]{2}{*}{$\begin{array}{l}p \\
\text { Value }\end{array}$} \\
\hline & HR (95\% CI) & & HR $(95 \% \mathrm{CI})$ & \\
\hline Gender & & 0.059 & & \\
\hline Male & 1 & & & \\
\hline Female & $0.442(0.19-1.03)$ & 0.059 & & \\
\hline Age (years) & & 0.777 & & \\
\hline$<60$ & 1 & & & \\
\hline$\geq 60$ & $1.078(0.643-1.806)$ & 0.777 & & \\
\hline Tumor location & & 0.412 & & \\
\hline Upper & 1 & & & \\
\hline Middle & $1.926(0.689-5.387)$ & 0.211 & & \\
\hline Lower & $1.582(0.525-4.768)$ & 0.415 & & \\
\hline Tumor length $(\mathrm{cm})$ & & 0.859 & & \\
\hline$<5$ & 1 & & & \\
\hline$\geq 5$ & $0.954(0.569-1.699)$ & 0.751 & & \\
\hline $\begin{array}{l}\text { Tumor } \\
\text { differentiation }\end{array}$ & & 0.424 & & \\
\hline Well & 1 & & & \\
\hline Moderate & $0.950(0.401-2.248)$ & 0.907 & & \\
\hline Poor & $1.415(0.548-3.653)$ & 0.474 & & \\
\hline $\begin{array}{l}\text { Pathological vessel } \\
\text { invasion }\end{array}$ & & 0.005 & & 0.169 \\
\hline Negative & 1 & & 1 & \\
\hline Positive & $2.356(1.289-4.304)$ & & $0.144(0.009-2.277)$ & 0.169 \\
\hline Pathologic T stage & & 0.363 & & \\
\hline $\mathrm{T} 1$ & 1 & & & \\
\hline $\mathrm{T} 2$ & $0.454(0.135-1.531)$ & 0.203 & & \\
\hline T3 & $0.698(0.213-2.282)$ & 0.552 & & \\
\hline $\mathrm{T} 4$ & $0.501(0.112-2.240)$ & 0.366 & & \\
\hline Pathologic N stage & & $<0.001$ & & 0.004 \\
\hline No & 1 & & 1 & \\
\hline N1 & $1.659(0.8-3.44)$ & 0.174 & $1.666(0.798-3.478)$ & 0.174 \\
\hline $\mathrm{N} 2$ & 3.803 (1.881-7.687) & $<0.001$ & $2.82(1.367-5.815)$ & 0.005 \\
\hline N3 & $4.281(1.970-9.304)$ & $<0.001$ & $3.882(1.757-8.581)$ & 0.001 \\
\hline $\begin{array}{l}\text { No. of lymph nodes } \\
\text { retrieved }\end{array}$ & & 0.003 & & 0.001 \\
\hline$\leq 20$ & 1 & & 1 & \\
\hline$<20 \sim \leq 30$ & $0.555(0.297-1.036)$ & 0.064 & $0.736(0.385-1.405)$ & 0.352 \\
\hline$>30$ & $0.299(0.151-0.594)$ & 0.001 & $0.301(0.150-0.602)$ & 0.001 \\
\hline Adjuvant therapy & & 0.687 & & \\
\hline None & 1 & & & \\
\hline Chemotherapy & $0.898(0.534-1.512)$ & 0.687 & & \\
\hline p-AKT1 & & $<0.001$ & & $<0.001$ \\
\hline Low-level & 1 & & 1 & \\
\hline High-level & $4.626(2.599-8.234)$ & $<0.001$ & $4.156(2.273-7.596)$ & $<0.001$ \\
\hline
\end{tabular}

Previous data demonstrate that in general, the more lymph nodes resected, the better the survival [31-32]. Using the SEER database, Schwarz et al. [33] examined lymph node data from over 5600 patients who underwent oesophagectomy and found that higher total lymph node counts were associated with better OS, independent of nodal status or histology. To obviate the inherent limitations of population-based studies previously discussed, Altorki et al. [34] recapitulated results obtained in large population studies demonstrating the association between total nodal count and survival in oesophageal cancer within the context of a single high-volume institution using standardized surgical techniques. The results suggested that a higher nodal count favourably influences survival after oesophagectomy. Even in node-negative patients, the benefit of survival was statistically significant when more than 40 nodes were removed. In the current study, we also investigated the effect of lymph node retrieval number on LPFS. Based on various data, it was found that optimum lymphadenectomy depends on T classification: For pT1-2, approximately 20 nodes and for pT3-4, 30 nodes or more. Therefore, we choose 20 and 30 nodes as the cutoff points for the number of lymph nodes retrieved in our study. Through univariable and multivariable Cox regression analysis, we identified the number of lymph nodes retrieved as independent prognostic factors for LPFS. As shown in Fig. 2C, a higher number of lymph nodes retrieved was associated with a better LPFS.

Several possible reasons for the impact of total retrieved lymph nodes on survival are presented. One reason is that it is likely that stage migration may occur in the context of more extensive node dissection. Also, retrieving more lymph nodes makes it more likely that the nodal sites containing micrometastatic disease will be cleared. Moreover, the number of retrieved nodes may reflect the adequacy of surgical, pathological, and institutional care, all of which tend to affect treatment outcomes [34-36]. Some studies reported that the lymph node metastatic ratio was also an independent predictor for OS in oesophageal carcinoma [37-38]. While in our study, we analysed the association of locoregional control with the number of lymph nodes retrieved other than the lymph node metastatic ratio. The lymph node metastatic ratio is determined by the number of positive lymph nodes (pathologic $\mathrm{N}$ stage) and total number of lymph nodes retrieved (the denominator). The prognostic value of lymph node metastatic ratio can be diluted because the denominator is variable [39]. Especially in node-negative patients, the predictive value of the number of lymph nodes 
retrieved might be superior to the lymph node metastatic ratio.

Several limitations of our study should be acknowledged. First, the current study used a retrospective design with a limited number of patients analysed. Second, the nomogram contained only one biomarker. More biomarkers should be analysed based on their association with different oncologic mechanisms in ESCC in future studies. The presence of multiple biomarkers might enhance the power of the nomogram for risk stratification. Furthermore, we did not perform an external validation, so concerns about generalizability are warranted. Therefore, the presented nomogram needs to be replicated and then prospectively validated before it can be implemented in clinical practice.

In conclusion, expression of p-AKT1, pathologic $\mathrm{N}$ category and number of lymph nodes retrieved were potential prognostic factors for LPFS in patients with ESCC after radical oesophagectomy. The nomogram integrated expression of p-AKT1 and clinicopathological factors, and could give rise to a new stratification system. It could be used to accurately predict locoregional recurrence in patients with ESCC (C-index=0.78) and help clinicians make better-informed individualized treatment recommendations.

\section{Acknowledgements}

This work was sponsored by the Scientific Research Foundation for the Returned Overseas Chinese Scholars, State Education Ministry, and Foundation of Shanghai Municipal Commission of Health and Family Planning (NO.201540211), and Shanghai Committee of Science and Technology, Natural Science Foundation of Shanghai, China (NO.15ZR1432100).

\section{Competing Interests}

The authors have declared that no competing interest exists.

\section{References}

1. Nakajima M, Kato $H$. Treatment options for esophageal squamous cell carcinoma. Expert Opin Pharmacother. 2013; 14: 1345-1354.

2. Mariette C, Balon JM, Piessen G, et al. Pattern of recurrence following complete resection of esophageal carcinoma and factors predictive of recurrent disease. Cancer. 2003; 97: 1616-1623.

3. Nakagawa S, Kanda T, Kosugi S, et al. Recurrence pattern of squamous cell carcinoma of the thoracic esophagus after extended radical esophagectomy with three-field lymphadenectomy. J Am Coll Surg. 2004; 198: 205-211.

4. Natsugoe S, Matsumoto M, Okumura H, et al. Clinical course and outcome after esophagectomy with three-field lymphadenectomy in esophageal cancer. Langenbecks Arch Surg. 2010; 395: 341-346.

5. Schreiber D, Rineer J, Vongtama D, et al. Impact of postoperative radiation after esophagectomy for esophageal cancer. J Thorac Oncol. 2010; 5: 244-250.

6. Tepper J, Krasna MJ, Niedzwiecki D, et al. Phase III trial of trimodality therapy with cisplatin, fluorouracil, radiotherapy, and surgery compared with surgery alone for esophageal cancer: CALGB 9781. J Clin Oncol. 2008; 26: 1086-1092.
7. Malthaner RA, Wong RKS, Rumble RB, et al. Neoadjuvant or adjuvant therapy for resectable esophageal cancer: a systematic review and meta-analysis. BMC Med. 2004; 2: 35.

8. Chen J, Zhu J, Pan J, et al. Postoperative radiotherapy improved survival of poor prognostic squamous cell carcinoma esophagus. Ann Thorac Surg. 2010; 90: 435-442.

9. Xiao ZF, Yang ZY, Miao YJ, et al. Influence of number of metastatic lymph nodes on survival of curative resected thoracic esophageal cancer patients and value of radiotherapy: Report of 549 cases. Int J Radiat Oncol Biol Phys. 2005; 62: 82-90.

10. Fareed KR, Kaye P, Soomro IN, et al. Biomarkers of response to therapy in oesophago-gastric cancer. Gut. 2009; 58: 127-143.

11. Lin DC, Du XL, Wang MR. Protein alterations in ESCC and clinical implications: a review. Dis Esophagus. 2009; 22: 9-20.

12. Zhu Z, Yu W, Fu X, et al. Phosphorylated AKT1 is associated with poor prognosis in esophageal squamous cell carcinoma. J Exp Clin Canc Res. 2015; 34: 1-8.

13. Zhu Z, Yu W, Li H, et al. Nodal skip metastasis is not a predictor of survival in thoracic esophageal squamous cell carcinoma. Ann Surg Oncol. 2013; 20: 3052-3058.

14. Zhu Z, Chen $\mathrm{H}, \mathrm{Yu} \mathrm{W}$, et al. Number of negative lymph nodes is associated with survival in thoracic esophageal squamous cell carcinoma patients undergoing three-field lymphadenectomy. Ann Surg Oncol. 2014; 21: 2857-2863.

15. Rice TW, Rusch VW, Ishwaran H, et al. Cancer of the esophagus and esophagogastric junction: data-driven staging for the seventh edition of the American Joint Committee on Cancer/International Union Against Cancer Staging Manuals. Cancer. 2010; 116: 3763-3773.

16. Skliris GP, Hube F, Gheorghiu I, et al. Expression of small breast epithelial mucin (SBEM) protein in tissue microarrays (TMAs) of primary invasive breast cancers. Histopathology. 2008; 52: 355-369.

17. Steyerberg EW, Eijkemans MJ, Harrell FE, et al.. Prognostic modeling with logistic regression analysis: in search of a sensible strategy in small data sets. Med Decis Making. 2001; 21: 45-56.

18. Kattan MW. Validating a prognosting model. Cancer. 2006; 107: 2523-2524.

19. Bhansali MS, Fujita H, Kakegawa T, et al. Pattern of recurrence after extended radical esophagectomy with three-field lymph node dissection for squamous cell carcinoma in the thoracic esophagus. World J Surg. 1997; 21: 275-281.

20. Courtney KD, Corcoran RB, Engelman JA. The PI3K pathway as drug target in human cancer. J Clin Oncol. 2010; 28: 1075-1083.

21. Manning BD, Cantley LC. AKT/PKB signaling: navigating downstream. Cell. 2007; 129: 1261-1274

22. Brunet A, Bonni A, Zigmond MJ, et al. Akt promotes cell survival by phosphorylating and inhibiting a Forkhead transcription factor. Cell. 1999; 96: 857-868.

23. Cai QQ, Deng HX, Xie D, et al. Phosphorylated AKT protein is overexpressed in human peripheral T-cell lymphomas and predicts decreased patient survival. Clin Lymphoma Myeloma Leuk. 2012; 12: 106-112.

24. Li R, Dai H, Wheeler T, et al. High levels of total Akt-1 predicts high-risk of biochemical recurrence and prostate cancer specific death. J Urology. 2009; 184(Suppl 4): 775-776.

25. Spears M, Cunningham CA, Taylor KJ, et al. Proximity ligation assays for isoform-specific Akt activation in breast cancer identify activated Akt1 as a driver of progression. J Pathol. 2012; 227: 481-489.

26. Kreisberg JI, Malik SN, Prihoda TJ, et al. Phosphorylation of AKT(Ser473) is an excellent predictor of poor clinical outcome in prostate cancer. Cancer Res. 2004; 64: 5232-5236.

27. Ayala G, Thompson T, Yang G, et al. High levels of phosphorylated form of Akt-1 in prostate cancer and non-neoplastic prostate tissues are strong predictors of biochemical recurrence. Clin Cancer Res. 2004; 10: 6572-6578.

28. Yang HX, Feng W, Wei JC, et al. Support vector machine-based nomogram predicts postoperative distant metastasis for patients with oesophageal squamous cell carcinoma. Br J Cancer. 2013; 109: 1109-1116.

29. Liu JS, Huang $Y$, Yang X, et al. A nomogram to predict prognostic values of various inflammatory biomarkers in patients with esophageal squamous cell carcinoma. Am J Cancer Res. 2015; 5: 2180-2189.

30. Hayashi Y, Xiao L, Suzuki A, et al. A nomogram associated with high probability of malignant nodes in the surgical specimen after trimodality therapy of patients with oesophageal cancer. Eur J Cancer. 2012; 48: 3396-3404.

31. Baba $Y$, Watanabe $M$, Shigaki $H$, et al. Negative lymph-node count is associated with survival in patients with resected esophageal squamous cell carcinoma. Surgery. 2013; 153: 234-241.

32. Cao JL, Yuan P, Wang LM, et al. Clinical nomogram for predicting survival of esophageal cancer patients after esophagectomy. Sci Rep 2016; [Epub ahead of print].

33. Schwarz RE, Smith DD. Clinical impact of lymphadenectomy extent in resectable esophageal cancer. J Gastrointest Surg. 2007; 11: 1384-1393.

34. Altorki NK, Zhou XK, Stiles B, et al. Total number of resected lymph nodes predicts survival in esophageal cancer. Ann Surg. 2008; 248: 221-226.

35. $\mathrm{Hu} \mathrm{Y,} \mathrm{Hu} \mathrm{C,} \mathrm{Zhang} \mathrm{H}$, et al. How does the number of resected lymph nodes influence TNM staging and prognosis for esophageal carcinoma? Ann Surg Oncol. 2010; 17: 784-790.

36. Izbicki JR, Hosch SB, Pichlmeier $U$, et al. Prognostic value of immunohistochemically identifiable tumor cells in lymph nodes of patients 
with completely resected esophageal cancer. N Engl J Med. 1997; 337: 1188-1194.

37. Wei C, Deng WY, Li N, et al. Lymph Node Ratio as an Alternative to the Number of Metastatic Lymph Nodes for the Prediction of Esophageal Carcinoma Patient Survival. Dig Dis Sci. 2015; 60: 2771-2776.

38. Hou X, Wei JC, Xu Y, et al. The Positive Lymph Node Ratio Predicts Long-Term Survival in Patients with Operable Thoracic Esophageal Squamous Cell Carcinoma in China. Ann Surg Oncol. 2013; 20: 1653-1659.

39. Kelty CJ, Kennedy CW, Falk GL. Ratio of Metastatic Lymph Nodes to Total Number of Nodes Resected is Prognostic for Survival in Esophageal Carcinoma. J Thorac Oncol. 2010; 5: 1467-1471. 